\title{
An Improved Technique for Bringing of the Buccally Second Molars to the Occlusion
}

\author{
Fatih Celebi* \\ Department of Orthodontics, University of Gaziosmanpasa, Turkey
}

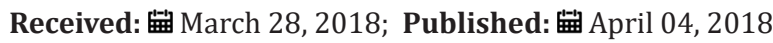

*Corresponding author: Fatih Celebi, Department of Orthodontics, Faculty of Dentistry, University of Gaziosmanpasa, 60100 Tokat, Turkey

\begin{abstract}
Buccally posioned teeth are parts of malocclusion which is commonly encountered in clinical cases. Treatment of these teeth can be difficult and time consuming, depending on their relationships to the counter teeth. In this pearl, we described that how the second molar tooth positioned at the scissor bite can be brought to the occlusion without leading to the handicaps.
\end{abstract}

Keywords: Buccally positioned teeth; Molar intrusion; Open bite

\section{Opinion}

Inclusion of the buccally positioned second molar teeth to the fixed treatment leads posterior dental expansion. When the expansion is occurring, palatal cusps of the buccal teeth tilt into the under the occlusal line. Especially in openbite or openbite tendency cases, this can make the situation worse. In this pearl, we described that how the second molar tooth positioned at the scissor bite can be brought to the occlusion without leading to the aforementioned handicaps. With this technique, the tooth is not only brought to the occlusion. In addition to that, sagged palatal cusps of the tooth can be intruded. Thus, since there will be no clashing of the cusps, there is no need for using the auxiliary bite opening mechanics while bringing.
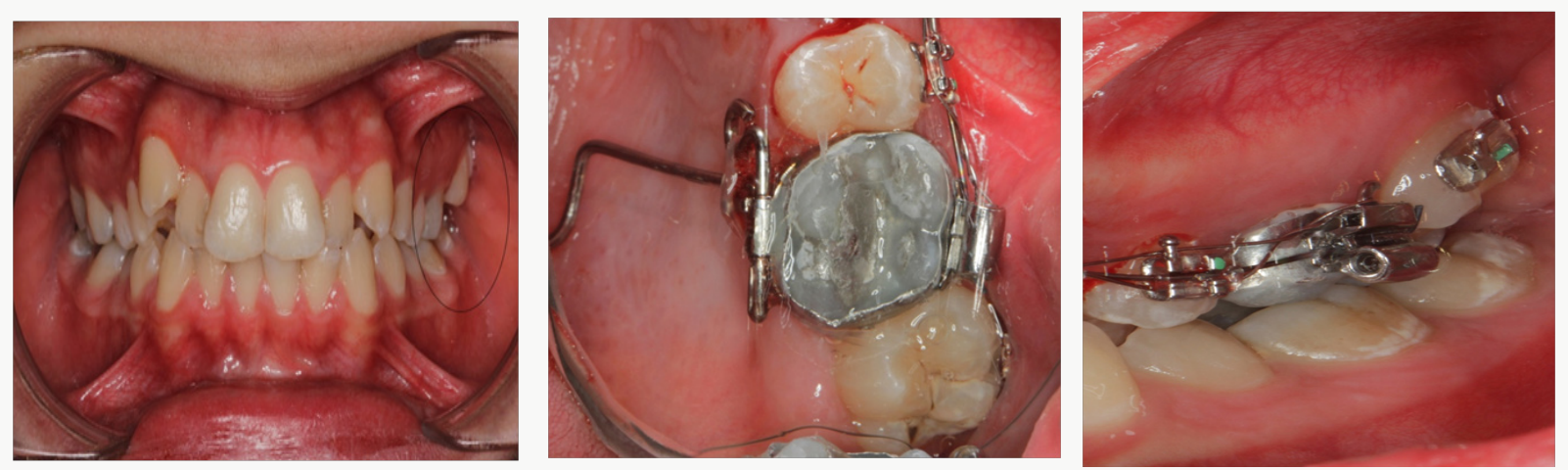

Figure 1A, 1B \& 1C: A female patient who had treatment planning with teeth extractions. Scissor bite can be observed at upper left second molar tooth.

Firstly, modified transpalatal arch (TPA) is blended. Modification is the adding of a swan shaped hook to the end of the TPA. Next, It placed to sheath of the upper first molar band. An attachment such as bondable tube or button is bonded to the upper second molar and a piece of elastomeric chain is stretched from the hook to the attachment. The technique was demonstrated in a female patient (Figure 1A, 1B \& 1C). Only 1 month later, you can notice the correction of the scissor bite (Figure 2A, 2B \& 2C). Final result was presented in Figure 3A and 3B. 

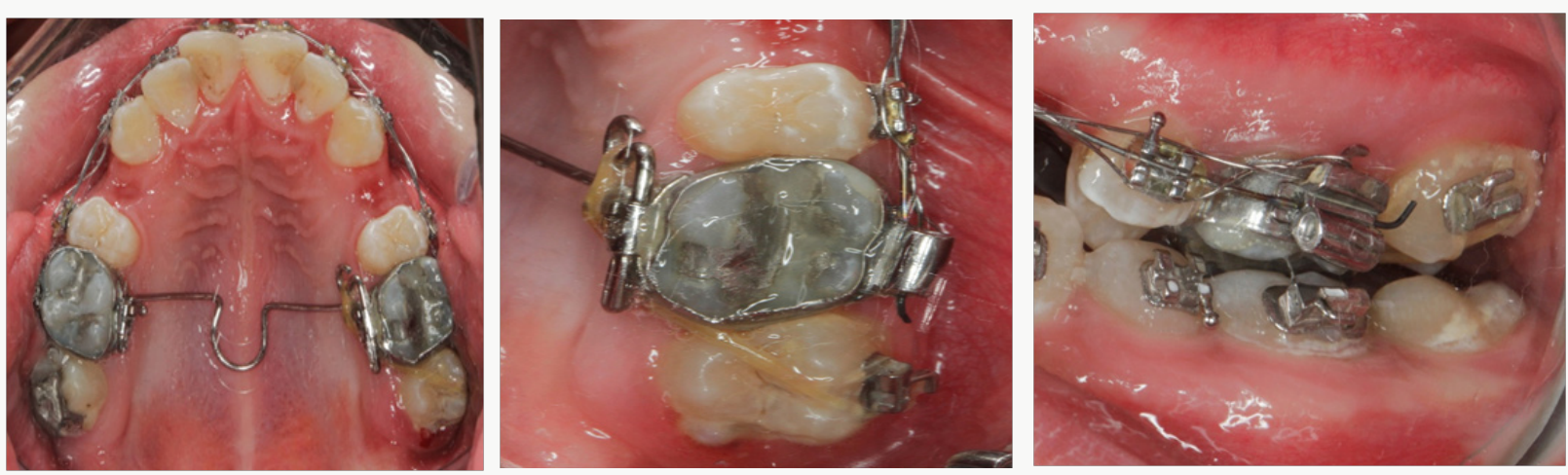

Figure 2A, 2B \& 2C: 1 month later after installation of the mechanics.
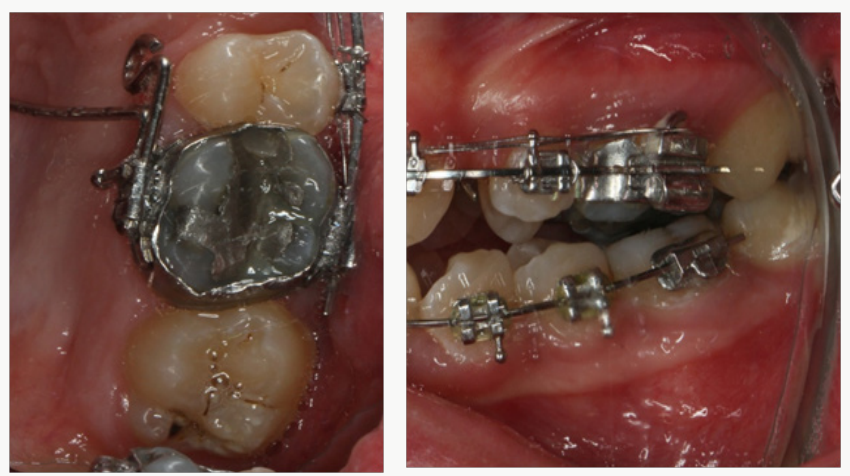

Figure 3A \& 3B: Correction of the scissor bite.

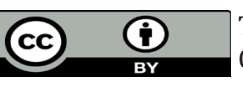

To Submit Your Article Click Here: Submit Article

This work is licensed under Creative Commons Attribution 4.0 License

DOI: 10.32474/MADOHC.2018.01.000125

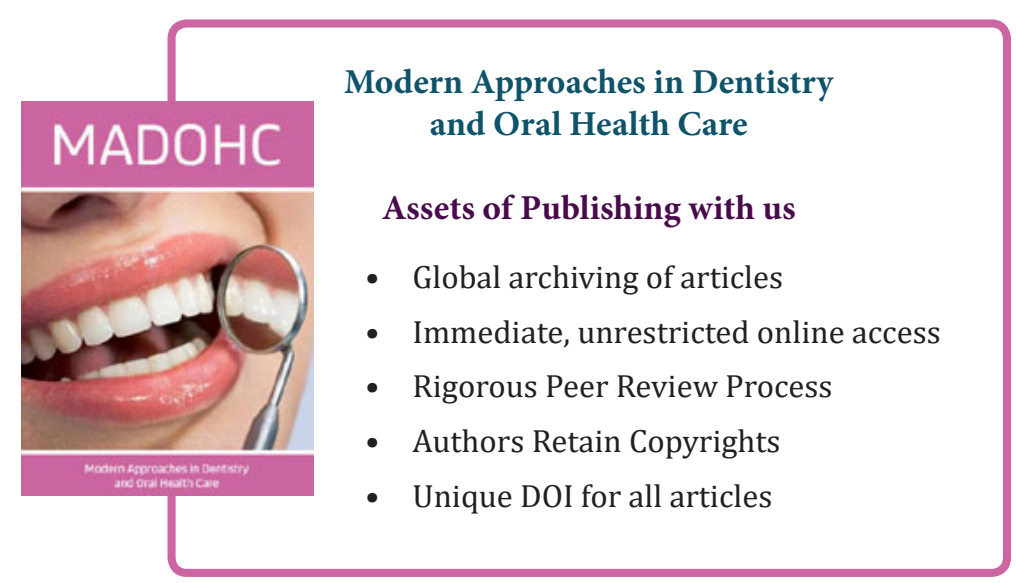

